\title{
An Antibody Fab Fragment-based Chimeric Antigen Receptor Could Efficiently Eliminate Human Thyroid Cancer Cells
}

\author{
Honghong Duan ${ }^{1}$, Huibin Huang ${ }^{2}$, Guangjun Jing ${ }^{3}$ \\ 1. Department of obstetrics and gynecology, The Second affiliated Hospital of Fujian Medical University, Quanzhou Fujian 362000, China. \\ 2. Department of Endocrinology, The Second affiliated Hospital of Fujian Medical University, Quanzhou Fujian 362000, China. \\ 3. iCATAB biomedical co. LTD, Suzhou 215000, China. \\ $\square$ Corresponding author: Huibin Huang, Department of Endocrinology, The Second affiliated Hospital of Fujian Medical University, NO.34 North Zhongshan \\ Road of Quanzhou, Fujian Province, P.R. China. Email:huibinhuang@aliyun.com; Phone: (86)13313872001; (86)0595-22770562 \\ (c) Ivyspring International Publisher. This is an open access article distributed under the terms of the Creative Commons Attribution (CC BY-NC) license \\ (https://creativecommons.org/licenses/by-nc/4.0/). See http://ivyspring.com/terms for full terms and conditions.
}

Received: 2018.09.24; Accepted: 2019.02.27; Published: 2019.04.21

\begin{abstract}
Thyroid cancer remains a significant health problem worldwide. Traditional chemotherapy does generate long-term benefit but are usually accompanied by severe side effects. Immunotherapy by adoptive infusion of $T$ cells is now an attractive alternative to chemotherapy. Chimeric antigen receptor engineered lymphocytes have produced tremendous clinical outcomes in treating leukemia or lymphoma, but not in solid tumors, which is in part due to the low affinity of single chain Fv fragment or the rapid loss of transfused T cells. In present research, we designed a novel Fab based chimeric antigen receptor, which inherits the advantages of Fab fragment as well as the natural TCR receptor. The novel Fab CAR could recognize the tumor antigens independent of $\mathrm{MHC} /$ peptide complex, and mimic the natural activation process of endogenous TCR, therefore extend the life span of CAR-engineered T cells and generate durable clinical effects.
\end{abstract}

Key words: CAR, Fab, CAR-T, thyroid cancer

\section{Introduction}

Adoptive $\mathrm{T}$ cell therapy using genetically engineered $\mathrm{T}$ cells expressing a chimeric antigen receptor (CAR) against tumor antigens is now a promising caner immunotherapeutic approach for leukemia and lymphoma.[1, 2] The CAR molecules consists of a single chain antibody fragment $(\mathrm{scFv})$ based ectodomain and an intracellular stimulatory domain. The $\mathrm{scFv}$ ectodomain confers the $\mathrm{T}$ cells responding to tumor cell surface antigens independent of MHC. The intracellular signaling domain influence $\mathrm{T}$ cell activity, persistence and efficacy. Incorporation of 4-1BB or CD28 in tandem with CD3ל showed improved clinical outcome.[3, 4] However, most scFvs still display the tendency of aggregation, [5] compromising the antigen-antibody interaction. Especially when the surfaced anchored $\mathrm{scFv}$ in the CAR aggregates, an unexpected signal will be transduced, leading to constitutively $\mathrm{T}$ cell activation in an antigen-independent mechanism, resulting in more rapid loss of the transferred T cells, and reduced effectiveness.[6] Moreover, the binding affinity and stability of scFv are inferior when compared with its Fab fragment, indicating not all scFvs are suitable for CAR construction and extra screening steps are necessary for screening a candidate scFvs for CAR.

The $\mathrm{T}$ cell receptor is heterodimer consisting of a- and $\beta$-chain, interacting with a complex of MHC and peptide. Either a or $\beta$ chain contains a variable domain and a constant domain. Each variable region have three complementarity determining regions, which reserve the specificity of TCR with its target $\mathrm{MHC}$ /peptide complex.[7]

There are published evidences indicating that the affinity of TCR determines $\mathrm{T}$ cell avidity and influences antigenic stimulation. $[8,9]$ The affinity of native TCR with its target $\mathrm{MHC} /$ peptide complex is 
relatively low for maintaining self-tolerance, generally ranging between 1 and $50 \mu \mathrm{M}$.[10] Although adoptively infusion of T-cell receptor (TCR)engineered lymphocytes possesses great potential for treatment of patients with advanced cancer, most immunotherapy-targeted tumor-associated antigens are non-mutated self-antigens and $\mathrm{T}$ cell responses against these self-antigens are generally weak and the affinity between TCR and MHC/peptide complex is suboptimal.[11] Consequently the use of TCRengineered $T$ cells for cancer treatment is limited. In present research we took CD276 as the target and designed a novel antibody Fab-based ectodomain in tandem with natural TCR signaling domains, this design could overcome the drawbacks of using $\mathrm{scFv}$ and took advantages of endogenous TCR signaling to mimic the activation of natural $\mathrm{T}$ cells.

\section{Materials and Methods}

\section{Development of monoclonal antibody against CD276}

The extracellular domain (29aa-466aa) of human CD276 (UniProtKB-Q5ZPR3) was codon optimized using GeneOptimizer from GeneArt and was synthesized by GenScript USA, followed by subcloning into $\mathrm{pET}-21 \mathrm{a}$ vector. The recombinant expression vector was transformed into BL21DE3 strain and single clone was randomly selected for expressing the recombinant protein. A Ni column was used for affinity purification of the His-tagged CD276 ECD protein. Five Balb/c mice was immunized with the recombinant CD276 protein after emulsion with Complete Freund's adjuvant or incomplete Freund's adjuvant. Hybridoma cells were prepared according to standard protocol using PEG1450 and SP2/0 cells as fusion partner.[12] After fusion, the positive clones were screened using a high-throughput flow cytometer (Thermo, Attune NXT flow cytometer). Finally the amino acids sequences of both heavy and light chains were obtained by hybridoma sequencing through standard procedures.[13] The binding of the murine monoclonal antibody was analyzed by flow cytometer and the affinity of the monoclonal antibody was determined by BiaCore T200.

\section{Design and construction of the lentiviral Fab-based chimeric antigen receptor}

As illustrated in figure 1, the heavy chain variable region and the first constant region were in tandem with the constant and intracellular signaling domain of natural TCR a chain while the light chain variable region and constant region were connected with the constant and intracellular signaling domain of natural TCR $\beta$ chain. The disulfide bond heavy and light chains was retained, which was the key to reserve the structure of antibody Fab fragment. As shown in figure 2, a signal peptide was included in both VH-CH1-Ca and VL-CL-C $\beta$. A self-cleavage peptide T2A was used to link these two ORFs. The full sequence was synthesized at GenScript after codon optimization and then subcloned into lentiviral vector. The lentiviral supernatant was produced using 293T cells, which were co-transfected with the gag/pol, VSVG and the lentiviral CAR vector using PEIMax. The lentivirus supernatant was harvested at 48 and $72 \mathrm{~h}$ post transfection, filtered through a $0.45 \mu \mathrm{m}$ filter and concentrated by ultracentrifugation for $2 \mathrm{~h}$ at $19,000 \mathrm{RPM}$. The lentivirus was stored at $-80^{\circ} \mathrm{C}$ for long term storage after aliquot. The concentrated lentivirus titer was determined by a qPCR method as described previously.[14]

\section{Donors and cell lines}

Peripheral blood mononuclear cells (PBMCs) were harvested from healthy volunteers with informed consent. Human thyroid cancer cell line FTC-133 and Jurkat were obtained from ATCC and were tested and authenticated by profiling polymorphic short tandem repeat markers. The firefly luciferase gene and full length of CD276 were stably inserted into the FTC-133 cells using lentivirus, and the recombinant stable FTC-133-luc-CD276 cells served as target cells for validating the Fab-based CAR-T cells.

\section{Preparation of Fab-based CAR-T cells}

Primary human CD3+ T cells were isolated from PBMCs by negative selection using a RosetteSep kit. The $\mathrm{T}$ cells were first activated by Dynabeads at a cell:bead ratio of 1:2 for 36 hours in serum-free medium X-Vivo 15 plus 300U/mL IL-2, 5ng/mL IL-7 and $10 \mathrm{ng} / \mathrm{mL}$ IL-15. The activated T cells were then transduced with lentivirus carrying Fab-CAR at a multiplicity of infection of 10 . The transduced T cells were maintained at a concentration of $5 \times 10^{5}$ cells $/ \mathrm{mL}$ in the serum-free $\mathrm{X}$-vio15 medium in the presence of IL-2, IL-7 and IL-15. The Fab-CAR T cells were continuously cultured for extra 10 days since lentivirus transduction.

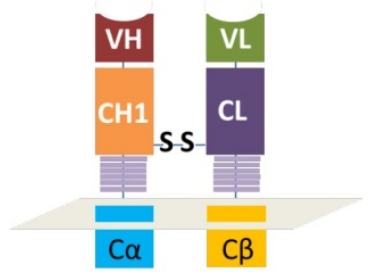

Figure 1. Schematically illustration of the antibody Fab-based chimeric antigen receptor. $\mathrm{VH}$ and $\mathrm{VL}$ represents the variable regions of heavy or light chains. $\mathrm{CH} 1$ means the first constant region of heavy chain and $\mathrm{CL}$ means the constant region of light chain. $C \alpha$ or $C \beta$ represents the orginal constant region and hige region of natural TCR. 
Figure 2. The elements of Fab-based chimeric antigen receptor. EFla promoter was employed to drive the expression of this CAR. Each ORF included a signaling peptide to direct the membrane anchor of this receptor. The two separate ORFs were in tandem with each other through a T2A peptide.

\section{Jurkat-Fab-CAR stable cells}

Jurkat cell line is a widely used cell model for investigating $\mathrm{T}$ cell function. Jurkat cells were transduced with the Fab-based CAR lentivirus and the stable Jurkat cells that constitutively express the CAR gene was screened by addition of $1 \mathrm{ug} / \mathrm{mL}$ puromycin into the culture medium. The recombinant FITC-conjugated CD276 extracellular protein was used to incubate with the resulted stable Jurkat cells. The membrane bound Fab CAR fragment was analyzed by flow cytometer.

\section{Cocultivation of Fab-CAR-T with thyroid cancer cells}

The target recombinant thyroid cancer FTC-133-Luc-CD276 cells were recovered from liquid nitrogen and was continuously subcultured until reaching logarithmic phase. Target tumor cells were resuspended at $5^{*} 10^{5}$ cell $/ \mathrm{mL}$ and $100 \mu \mathrm{L}$ of target cell suspension was inoculated into each well of a 96-well plate; the 96 well plate with target cells was incubated in a $5 \% \mathrm{CO}_{2}, 37^{\circ} \mathrm{C}$ incubator overnight. CAR-T cells or non-transduced control $\mathrm{T}$ cells were harvested by centrifugation at $250 \mathrm{xg}$ for $10 \mathrm{~min}$ at room temperature and resuspended with RPMI640 medium supplemented with $10 \%$ FBS; the culture medium was aspirated from the 96 well plate with target tumor cells and CAR-T cells were added into each well according to the $\mathrm{E} / \mathrm{T}$ ratio as indicated in the figure; the final volume was brought to $200 \mathrm{uL} /$ well using RPMI1640 with 10\% FBS. Each data point was repeated in triplicate. Six wells without addition of any $\mathrm{T}$ cells were reserved for Maxi lysis and Mini lysis. The plate was placed in $5 \% \mathrm{CO}_{2} 37^{\circ} \mathrm{C}$ incubator for 18 hours. After cultivation, a lysis buffer was added into the three wells of Maxi lysis. The plate was centrifuged at $1200 \mathrm{xg}$ for $5 \mathrm{~min}$ at room temperature. $100 \mu \mathrm{L}$ of supernatant was transferred into a new 96 well plate for determination of the secretion level of IL-2 and IFN- $\gamma$. ONEGlo reagent was added into the 96 well plate to investigate the luciferase activity. The percent lysis of target tumor cells by CAR-T was calculated by the formulation below. All reagents and cell culture materials were obtained from Thermo unless otherwise stated.

$$
\text { Lysis } \%=\left(1-\frac{R L U_{\text {Maxi }}-R L U_{\text {Sample }}}{R L U_{\text {Maxi }}-R L U_{\text {Min }}}\right) x 100 \%
$$

\section{Results}

\section{Characterization of the monoclonal antibody against CD276}

The recombinant thyroid cancer FTC-133-CD276 cells were stained with the selected monoclonal antibody and PE-conjugated secondary antibody. Flow cytomertry analysis showed that the selected monoclonal antibody could specifically bind surface CD276 protein on the recombinant FTC-133 cells (Figure 3). For quantifying the affinity, the recombinant CD276 protein was conjugated on a BiaCore T200 chip through amino coupling. The monoclonal antibody prepared from ascites was used as mobile phase. As shown in figure 4, the Ka was 3.15 $\mathrm{X} 10^{5} \mathrm{M}^{-1} \mathrm{~s}^{-1}$, kd was $0.003121 \mathrm{~s}^{-1}$ and the KD was 6.48 $\mathrm{X} 10^{-9} \mathrm{M}$.

\section{The interaction of membrane bound Fab-CAR with CD276 protein}

For determining whether the membrane anchored Fab-CAR could correctly bind CD276 protein, a stable Jurkat cells were transduced with lentivirus carrying the Fab-CAR. After cultivation under the selective pressure with puromycin, the resulted stable cells were incubated with a FITC-conjugated recombinant CD276 protein followed by flow cytometer analysis. As shown in figure 5, the membrane bound Fab-CAR could bind recombinant CD276 protein, indicating the novel CAR design could correctly form functional antibody Fab fragment.

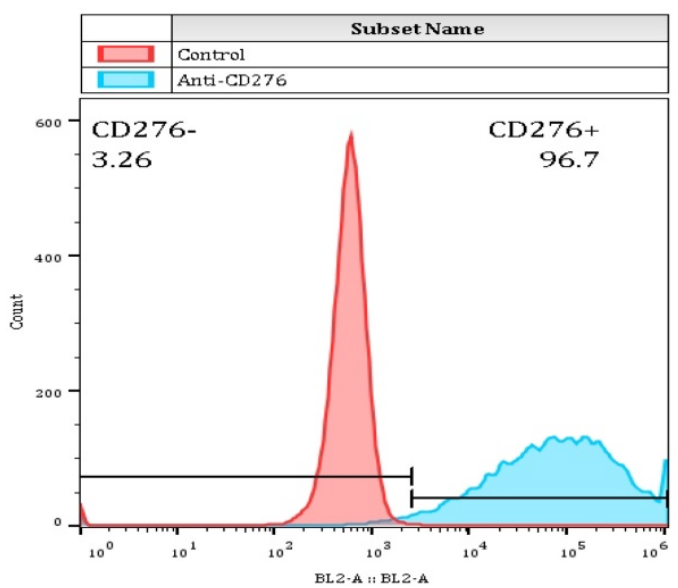

Figure 3. The binding capability of selected murine monoclonal antibody with CD276 was determined by flow cytometer. 0.5 ug mouse monoclonal antibody was incubated with $1 * 10^{6}$ FTC-133-CD276 stable cells followed by PE-conjugated secondary antibody staining. The samples were subjected to analysis by thermo Attune NXT flow cytometer. 


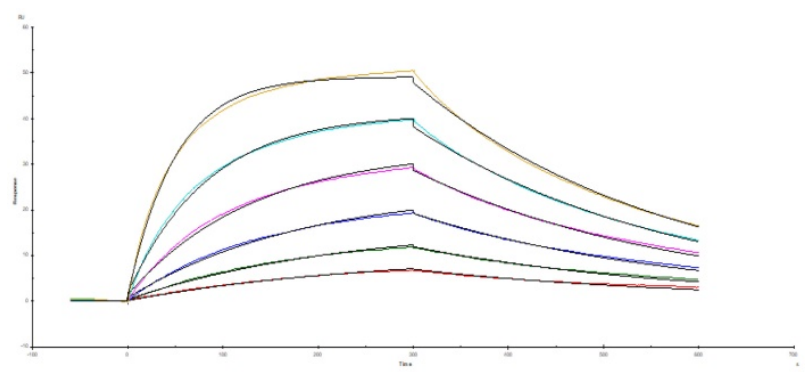

Figure 4. Affinity analysis of the monoclonal antibody. The affinity of the monoclonal antibody against $\mathrm{CD} 276$ was determined by BiaCore T200. The $\mathrm{Ka}$ was $3.15 \times 10^{5} \mathrm{M}^{-1} \mathrm{~s}^{-1}$, $\mathrm{kd}$ was $0.003121 \mathrm{~s}^{-1}$ and the $\mathrm{KD}$ was $6.48 \times 10^{-9} \mathrm{M}$.

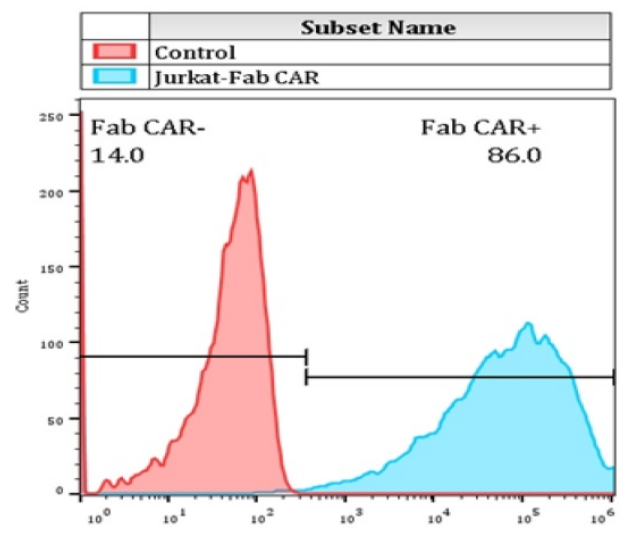

Figure 5. Flow cytometry analysis of surface anchored Fab-CAR on Jurkat stable cells. The surface expression of Fab-CAR was evaluated by flow cytometer after stained with FITC-conjugated CD276 protein. The data showed that the membrane bound Fab CAR could recognize the recombinant CD276 protein properly.

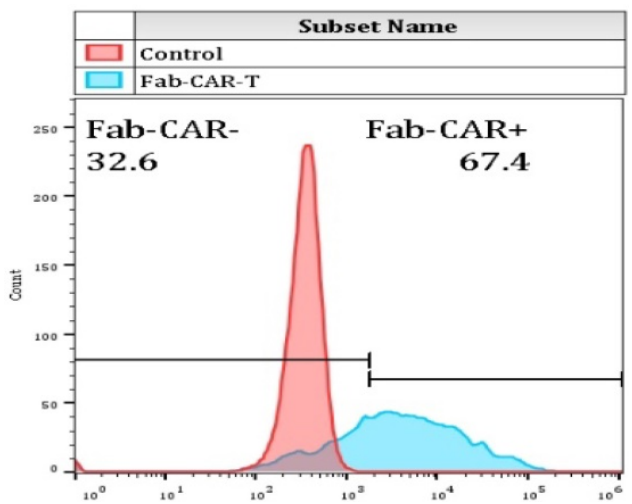

Figure 6. Flow cytometry analysis of surface anchored Fab-CAR on primary $\mathbf{T}$ cells after lentivirus transduction. The surface expression of Fab-CAR was evaluated by flow cytometer after stained with FITC-conjugated CD276 protein. The data showed that the membrane bound Fab CAR could recognize the recombinant $C D 276$ protein properly.

\section{The functional evaluation of Fab CAR}

For confirming whether the novel Fab CAR design could transduce signals upon engagement with its targeting tumor antigen, primary $\mathrm{T}$ cells isolated from healthy donors were transduced with the lentivirus carrying the novel Fab CAR gene. After confirmation of the surface expression of the Fab CAR by flow cytometer (Figure 6), the Fab-CAR-T cells were incubated with a targeted recombinant FTC-133 cells, which constitutively expressed firefly luciferase and CD276. After 18 hours co-cultivation, the supernatant was harvested for analyzing cytokine secretion such as IL2 and IFN- $\gamma$, the firefly luciferase activity was determined by using ONEGlo reagent. As shown in figure 7, the Fab-CAR $\mathrm{T}$ cells could efficiently and robustly eliminate targeted tumor cells as reflected by luciferase activity, when compared with the untransduced $\mathrm{T}$ cells, although the nonspecific lysis of tumor cells by parental $\mathrm{T}$ cells was apparent. The data in figure 8 showed that Fab CAR-T cells could robustly secret both IL-2 and IFN- $\gamma$ after being engaged with target tumor cells and the cytokine secretion level by CAR-T cells was much higher than control $\mathrm{T}$ cells. The control $\mathrm{T}$ cells barely secreted IFN- $\gamma$.

\section{Discussion}

The traditional chimeric antigen receptor is based on a single chain antibody fragment, in tandem with intracellular signaling domains. This traditional CAR design in early clinical data have demonstrated the tremendous potential of redirected $\mathrm{T}$ cells in the control of leukemia or lymphoma.[15-17] However, there are still many unknown problems why the traditional CAR-T cells have been less effective at inducing complete tumor responses and have mediated a high rate of on-target but off-tumor toxicity.[18-21] Choosing the proper tumor-associated antigen or tumor-specific antigen to target is a prerequisite for successful use of CAR-T. In a previous clinical trial, targeting ERBB2 with CAR T cells led to pulmonary toxicity and multi-organ failure and subsequent death due to the unexpected CAR T-cell recognition of low-level HER2 expression on the lung epithelium.[22] In present research, we took CD276 as the target antigen, as aberrant expression of B7-H3 was a promising immune checkpoint target for multiple cancer immunotherapy approaches.[23] We developed a new murine monoclonal antibody that could specifically bind CD276 at high affinity (nM). The $1^{\text {st }}$ generation of CAR designs only incorporated $\mathrm{CD} 3 \zeta$ as the intracellular signaling domain. However, the $1^{\text {st }}$ generation CARs activated the $\mathrm{T}$ cells suboptimally and failed clinical trials.[24] The $2^{\text {nd }}$ generation CARs included CD28 or 4-1BB costimulatory signaling domains to enhance T-cell activation, persistence, and antitumor efficacy. The third generation CAR designs incorporated two costimulatory signaling domains and has been used in solid tumors, but with only modest clinical responses. One of the reasons for this unsatisfying clinical results might be the relative lower affinity of $\mathrm{scFv}$ and the 
rapid loss of CAR-T in patient peripheral blood after CAR-T infusion. For overcoming this drawbacks, we designed a novel chimeric antigen receptor that incorporated the antibody Fab fragment in tandem with natural TCR intracellular signaling domain. And our data showed that this new design could function properly. The primary $\mathrm{T}$ cells bearing the membrane Fab chimeric antigen receptor could dissolve tumor cells efficiently and meanwhile robustly secret cytokines like IL2 and IFN-ү. This novel design combine the advantages of both antibody and natural TCR signaling domains. The stability and affinity of Fab fragment are superior to single chain Fv fragment, using the Fab instead of scFv could completely avoid the issue of aggregation of $\mathrm{scFv}$ and retains the high affinity of antibody to its antigen. Using TCR intracellular signaling domain instead of $4-1 \mathrm{bb}$ or CD28 could mimic the innate process of $\mathrm{T}$ cell activation and the strength of activation by this strategy is not as strong as that by $4-1 \mathrm{BB}$ or CD28, and could extend the life span of Fab CAR-T cells, which is a key factor for generation of durable clinical effects.

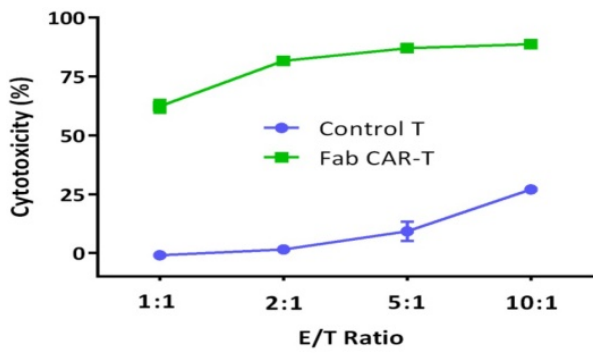

Figure 7. Cytotoxicity of Fab-CAR T cells. The lysis of targeted tumor cells FTC-133 was reflected by luciferase activity after cocultivation with Fab-CAR T cells at different $E / T$ ratio.
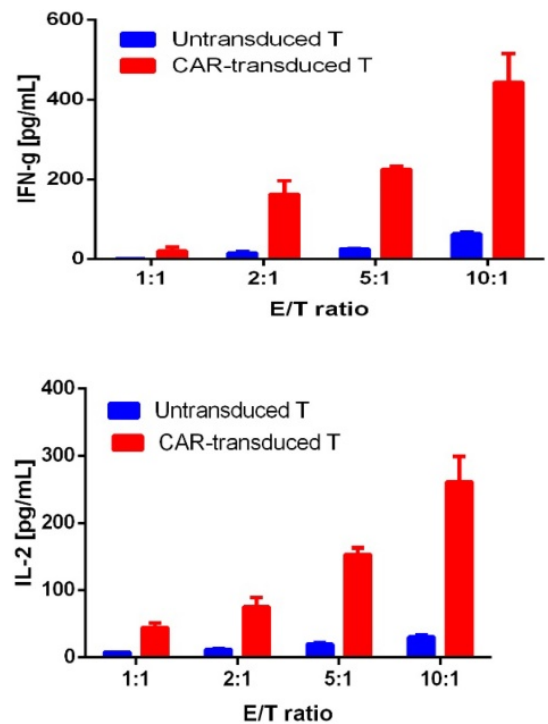

Figure 8. Cytokine release by Fab CAR-T cells. The secretion of cytokines, IL2 and IFN- $\gamma$, were investigated by ELISA after co-culturing with targeted tumor cells. The supernatant was harvested after 18 hours incubation. Both cytokines were secreted by tumor-activated Fab-CAR-T cells.

\section{Conclusions}

The novel Fab CAR could recognize the tumor antigens independent of MHC/peptide complex, and mimic the natural activation process of endogenous TCR, therefore extend the life span of CARengineered $\mathrm{T}$ cells and generate durable clinical effects.

\section{Abbreviations}

CAR: chimeric antigen receptor; scFv: single chain antibody fragment; PBMCs: Peripheral blood mononuclear cells; MHC: main histocompatibility complex; CAR-T: chimeric antigen receptor T-cell; Fab: fragment of antigen binding.

\section{Acknowledgements}

This work was supported by the National Natural Science Foundation of China (81370886), Science and technology project of Quanzhou Fujian (2018C044R), and Innovative medical research project of Fujian Province (2018-CX-33).

\section{Authors' contributions}

Huibin Huang conceived the idea and wrote the manuscript. Honghong Duan, Guangjun Jing performed the experiments and helped with the analysis of the results. All authors read and approved the final manuscript.

\section{Availability of data and materials}

Data generated or analysed during this study are included in this published article and are available from the corresponding author on reasonable request.

\section{Competing Interests}

The authors have declared that no competing interest exists.

\section{References}

1. Brentjens RJ, Riviere I, Park JH, Davila ML, Wang X, Stefanski J, Taylor C, Yeh $\mathrm{R}$, Bartido S, Borquez-Ojeda O, et al: Safety and persistence of adoptively transferred autologous CD19-targeted $\mathrm{T}$ cells in patients with relapsed or chemotherapy refractory B-cell leukemias. Blood 2011, 118:4817-4828.

2. Porter DL, Levine BL, Kalos $\mathrm{M}$, Bagg A, June $\mathrm{CH}$ : Chimeric antigen receptor-modified T cells in chronic lymphoid leukemia. N Engl J Med 2011, 365:725-733.

3. Kowolik CM, Topp MS, Gonzalez S, Pfeiffer T, Olivares S, Gonzalez N, Smith DD, Forman SI, Jensen MC, Cooper LJ: CD28 costimulation provided through a CD19-specific chimeric antigen receptor enhances in vivo persistence and antitumor efficacy of adoptively transferred T cells. Cancer research 2006, 66:10995-11004.

4. Song D-G, Ye Q, Carpenito C, Poussin M, Wang L-P, Ji C, Figini M, June CH, Coukos G, Powell DJ: In vivo persistence, tumor localization, and antitumor activity of CAR-engineered $\mathrm{T}$ cells is enhanced by costimulatory signaling through CD137 (4-1BB). Cancer research 2011, 71:4617-4627.

5. Gil D, Schrum AG: Strategies to stabilize compact folding and minimize aggregation of antibody-based fragments. Advances in bioscience and biotechnology (Print) 2013, 4:73.

6. Harris DT, Kranz DM: Adoptive T cell therapies: a comparison of $\mathrm{T}$ cell receptors and chimeric antigen receptors. Trends in pharmacological sciences 2016, 37:220-230 
7. Rudolph MG, Stanfield RL, Wilson IA: How TCRs bind MHCs, peptides, and coreceptors. Annu Rev Immunol 2006, 24:419-466.

8. Davis MM, Boniface JJ, Reich Z, Lyons D, Hampl J, Arden B, Chien Y-h: Ligand recognition by a $\beta \mathrm{T}$ cell receptors. Annual review of immunology 1998, 16:523-544.

9. Merwe PAvd, Davis SJ: Molecular interactions mediating $\mathrm{T}$ cell antigen recognition. Annual review of immunology 2003, 21:659-684.

10. Rudolph MG, Luz JG, Wilson IA: Structural and thermodynamic correlates of $\mathrm{T}$ cell signaling. Annual review of biophysics and biomolecular structure 2002, 31:121-149.

11. De Visser KE, Schumacher TN, Kruisbeek AM: CD8+ T cell tolerance and cancer immunotherapy. J Immunother 2003, 26:1-11.

12. Wood J: Immunization and fusion protocols for hybridoma production. In Proteins. Springer; 1984: 261-270

13. Koren S, Kosmač M, Venturini AC, Montanič S, Šerbec VČ: Antibody variable-region sequencing as a method for hybridoma cell-line authentication. Applied microbiology and biotechnology 2008, 78:1071-1078.

14. Barczak W, Suchorska W, Rubiś B, Kulcenty K: Universal real-time PCR-based assay for lentiviral titration. Molecular biotechnology 2015, 57:195-200.

15. Maude SL, Frey N, Shaw PA, Aplenc R, Barrett DM, Bunin NJ, Chew A, Gonzalez VE, Zheng Z, Lacey SF: Chimeric antigen receptor $\mathrm{T}$ cells for sustained remissions in leukemia. New England Journal of Medicine 2014, 371:1507-1517.

16. Kalos M, Levine BL, Porter DL, Katz S, Grupp SA, Bagg A, June CH: T cells with chimeric antigen receptors have potent antitumor effects and can establish memory in patients with advanced leukemia. Science translational medicine 2011, 3:95ra73-95ra73.

17. Cao J, Wang G, Cheng H, Wei C, Qi K, Sang W, Zhenyu L, Shi M, Li H, Qiao J: Potent anti-leukemia activities of humanized CD19-targeted Chimeric antigen receptor $\mathrm{T}$ (CAR-T) cells in patients with relapsed/refractory acute lymphoblastic leukemia. American journal of hematology 2018.

18. Lamers CH, Sleijfer S, Vulto AG, Kruit WH, Kliffen M, Debets R, Gratama JW, Stoter G, Oosterwijk E: Treatment of metastatic renal cell carcinoma with autologous T-lymphocytes genetically retargeted against carbonic anhydrase IX: first clinical experience. Journal of Clinical Oncology 2006, 24:e20-e22.

19. Kershaw MH, Westwood JA, Parker LL, Wang G, Eshhar Z, Mavroukakis SA, White DE, Wunderlich JR, Canevari S, Rogers-Freezer L: A phase I study on adoptive immunotherapy using gene-modified $\mathrm{T}$ cells for ovarian cancer. Clinical Cancer Research 2006, 12:6106-6115.

20. Park JR, DiGiusto DL, Slovak M, Wright C, Naranjo A, Wagner J, Meechoovet HB, Bautista C, Chang W-C, Ostberg JR: Adoptive transfer of chimeric antigen receptor re-directed cytolytic $\mathrm{T}$ lymphocyte clones in patients with neuroblastoma. Molecular therapy 2007, 15:825-833.

21. Parkhurst MR, Yang JC, Langan RC, Dudley ME, Nathan D-AN, Feldman SA, Davis JL, Morgan RA, Merino MJ, Sherry RM: T cells targeting carcinoembryonic antigen can mediate regression of metastatic colorectal cancer but induce severe transient colitis. Molecular Therapy 2011, 19:620-626.

22. Morgan RA, Yang JC, Kitano M, Dudley ME, Laurencot CM, Rosenberg SA: Case report of a serious adverse event following the administration of T cells transduced with a chimeric antigen receptor recognizing ERBB2. Molecular Therapy 2010, 18:843-851.

23. Zang X, Allison JP: The B7 family and cancer therapy: costimulation and coinhibition. Clinical cancer research 2007, 13:5271-5279.

24. Curran KJ, Pegram HJ, Brentjens RJ: Chimeric antigen receptors for T cell immunotherapy: current understanding and future directions. The journal of gene medicine 2012, 14:405-415. 